\title{
Polarimetric multi-frequency observations of a complete sample of radio sources
}

\author{
Vincenzo Galluzzi ${ }^{* a b}$ Marcella Massardi $^{b}$ and Loretta Gregorini ${ }^{b}$ \\ ${ }^{a}$ Dipartimento di Fisica e Astronomia, Università di Bologna \\ via Ranzani 1, Bologna, 40126, Italy \\ ${ }^{b}$ INAF, Osservatorio di Radioastronomia \\ Via Gobetti 101, Bologna, 40129, Italy \\ E-mail: vgalluzzieira.inaf.it, massardieira.inaf.it
}

\begin{abstract}
The polarisation properties of extragalactic radio sources at frequencies higher than $20 \mathrm{GHz}$ are still poorly constrained. Extending such characterization to higher frequencies would provide invaluable information about the physics of the emission processes, mostly dominated by selfabsorbed synchrotron emissions from Doppler boosted knot-like structures, closer and closer to the active nucleus. It is crucial to investigate the contribution to the polarised CMB signal by foreground sources, since they represent the dominant contaminant between 70 and $100 \mathrm{GHz}$ and up to angular scales of few arcminutes, hence being an issue for many experiments trying to detect primordial B-modes.

Thanks to new ATCA (Australia Telescope Compact Array) observations (September 2014) on a complete sample of 53 sources of the faint $\left(S_{20 \mathrm{GHz}}>200 \mathrm{mJy}\right)$ PACO (Planck-ATCA Co-eval Observations) sample, we have reconstructed the source polarisation spectral behaviour from 5.5 up to $38 \mathrm{GHz}$, and to deeper flux density levels than available so far, i.e. $\simeq 1 \mathrm{mJy}$. Preliminary analysis indicates a tiny trend of the fractional polarisation with frequency for steep sources and no significant trend with source flux density. It looks clear that polarisation properties cannot be simply inferred from total intensity ones, as the spectral properties are different in most of the cases, probably as a consequence of emission from different source components.
\end{abstract}

EXTRA-RADSUR2015 (*)

20-23 October 2015

Bologna, Italy

(*) This conference has been organized with the support of the Ministry of Foreign Affairs and International Cooperation, Directorate General for the Country Promotion (Bilateral Grant Agreement ZA14GR02 - Mapping the Universe on the Pathway to SKA)

\footnotetext{
* Speaker.
} 


\section{The state-of-the-art in polarimetric observations}

Emissions from bright extragalactic radio sources $\left(S_{20 \mathrm{GHz}} \sim 100 \mathrm{mJy}\right)$ are usually dominated by self-absorbed synchrotron from Doppler boosted knot-like structures closer and closer (as frequency increases) to the base of the jet in an active galactic nucleus (AGN).

The polarisation properties of extragalactic radio sources at frequencies higher than $20 \mathrm{GHz}$ are still poorly constrained: polarimetry generally requires higher sensitivities and a better knowledge of instrumental properties to perform accurate calibration of the observations. Other complications (in common with total intensity observations at higher frequencies) are that synchrotron emitting objects are expected to be fainter and more variable as frequency increases. Thus, our current knowledge at frequencies higher than $20 \mathrm{GHz}$ is based on few high frequency-selected samples that are typically bright and/or spectrally flat (for a more complete picture see [1]). From the analysis performed by Massardi et al. [2] of a complete sample of bright $\left(S_{20 \mathrm{GHz}}>500 \mathrm{mJy}\right)$ objects drawn from the Australia Telescope $20 \mathrm{GHz}$ (AT20G) survey in the $5-40 \mathrm{GHz}$ frequency range, it seems to emerge that the spectral behavior in polarisation is different from that in total intensity for several sources. The median polarisation fraction is $\simeq 2.5 \%$ at $20 \mathrm{GHz}$. However, this study does not show any statistically relevant trend of the polarisation fraction with the frequency nor with the flux density, meanwhile Sajina et al. [3], analysing 159 AT20G objects (with $S_{20 \mathrm{GHz}}>40 \mathrm{mJy}$ ) in the same frequency range, reported median polarisation fractions typically $<5 \%$ and increasing with frequency.

The Planck-ATCA Co-Eval Observations (PACO project $[4,5]$ ) were carried out with ATCA in the $4.5-40 \mathrm{GHz}$ frequency range. It observed 464 AT20G objects in 65 epochs between July 2009 and August 2010. What we present here is the polarimetric follow-up of a complete sample drawn from the PACO project catalogue.

\section{Polarimetric follow-up of PACO sources}

In September 2014 high sensitivity polarimetric observations $(\sigma \simeq 0.2 \mathrm{mJy})$ were obtained with ATCA (PI: Massardi, project C2922) in three sets of $2 \times 2 \mathrm{GHz}$ (namely 5.5 - 9, $18-24$ and $33-38 \mathrm{GHz})$. We observed 53 extragalactic sources drawn from the faint $\left(S_{20 \mathrm{GHz}}>200 \mathrm{mJy}\right)$ PACO sample, covering the southern ecliptic pole region (ecliptic latitude $<-75^{\circ}$ ). Effective spatial resolution ranges from 36 to 5 arcsec, considering only 5 of the 6 antennas, and between 4 and $0.5 \mathrm{arcsec}$ if also the sixth and furthest antenna is taken into account. The detection rate in polarisation is about $90 \%$ at $5 \sigma$ and intra-band depolarisation (expected higher at lower frequencies) does not indeed appreciably affect our polarisation flux density measurements.

Highly accurate fitting spectra are obtained for 50 of the 53 objects both in total intensity and in polarisation (some of them are shown as magenta and blue solid lines in Fig. 1). Almost all of the spectra follow a double power law model (in agreement with PACO results [5]). However, there are 2 sources in total intensity and other 2 in polarisation which depart from this description. We use flux densities from fitting to classify spectra both in total intensity and polarisation, by considering the spectral indices in two frequency intervals, namely $5.5-10 \mathrm{GHz}$ and $18-28 \mathrm{GHz}$, applying criteria reported in [2]. Indeed, already by an intuitive distinction in flat, steep and peaked spectra, it is possible to see that several sources behave differently in polarisation with respect to 
total intensity: e.g., the distribution of spectral behaviours in polarisation for steep sources (34 in our case) seems to be bimodal with more peaked objects (15) than steep ones (11). Since a steepening in all the spectra occurs for frequencies $\gtrsim 30 \mathrm{GHz}$ (as reported by PACO project), it is also worth noting the small number of flat behaviours (only 3 ) in the $5-40 \mathrm{GHz}$ frequency range.

By considering the polarisation fraction, we report a median value $\simeq 2.1 \%$ at $18 \mathrm{GHz}$ (in agreement with $[6,2])$ higher for steep spectra $(\simeq 2.4 \%)$ than for peaked ones $(\simeq 1.6 \%)$. Flat sources are too few to perform any statistical analysis. For our sample there is no indication of a trend of the polarisation fraction with the flux density at any observed frequency. To investigate any trend with the latter, we analyse distributions of the polarisation fraction at the different frequencies: given the high detection rate in polarisation $(\simeq 90 \%$ and quite homogeneous across the frequency range) we can assess a low detection bias level. We repeat the scheme by considering steep and peaked spectra alone. Since these distributions are not symmetric, we use median and inter-quartiles values to describe them. A statistically significant indication of a slightly increasing trend seems to emerge for steep sources only. In fact, the difference between the polarisation fractions at $38 \mathrm{GHz}$ and $5.5 \mathrm{GHz}$ is $(0.3 \pm 0.3) \%$ for all the sources and $(0.7 \pm 0.4) \%$ for steep ones. Thus, the need arises to both enlarge the sample and the spectral coverage to better characterize the behaviour of the polarisation fraction. For this purpose, ATCA observations are scheduled at the end of March 2016 (PI: Massardi) for 106 sources in the $2-40 \mathrm{GHz}$ frequency range, and 31 sources already observed in September 2014 are included in an ALMA-Cycle 3 project (approved with high-ranking, PI: Galluzzi) at $\simeq 100 \mathrm{GHz}$.
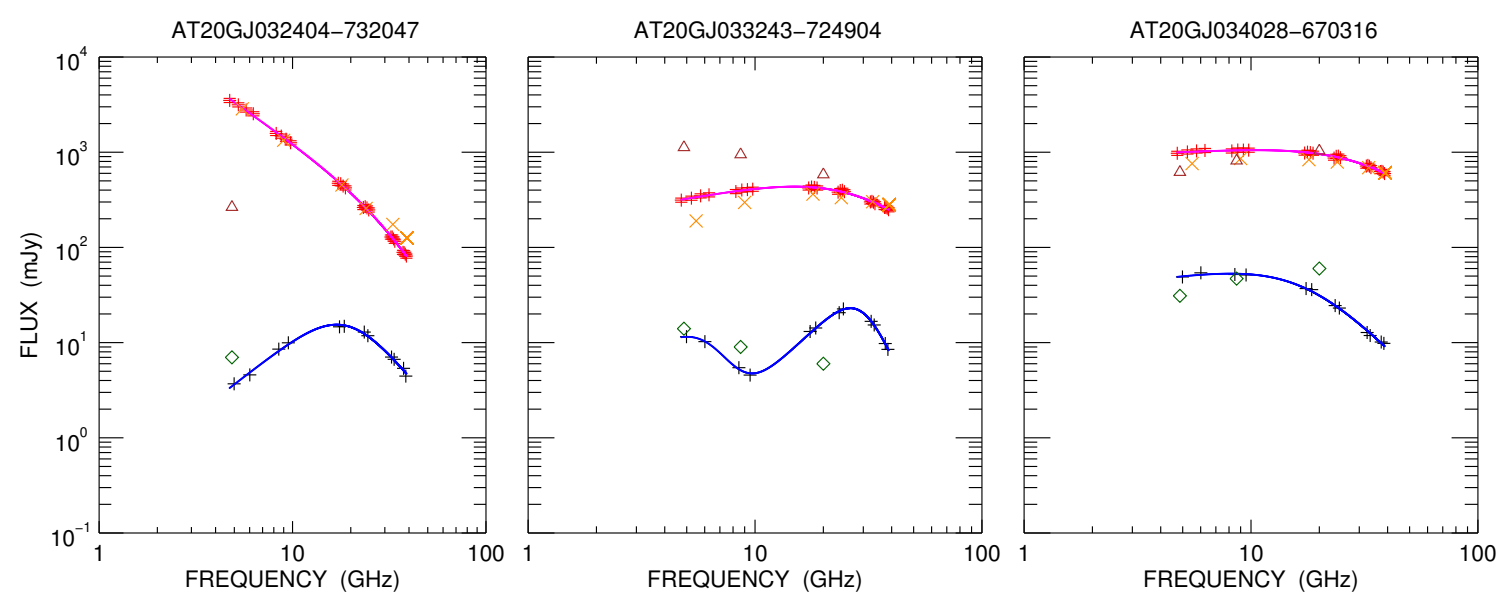

Figure 1: Spectra in total intensity and polarisation (error bars are not displayed since they are smaller than plotted symbols) for some sources drawn from the 53 of the faint PACO sample. Total intensity: red crosses indicate total intensity ATCA September 2014 observations (each point represents a 512 MHz-bin) and magenta solid lines indicate fitting curves. Median PACO flux densities (July 2009-August 2010) are indicated with orange " $x$ ". AT20G observations (best epoch in 2004-2008) are reported with brown triangles. Polarisation: black crosses indicate September 2014 observations (each point represents a $1 \mathrm{GHz}$-bin and the $2 \mathrm{GHz}$-full bandwidth, respectively) and blue solid lines indicate fitting curves. AT20G observations (best epoch in 2004-2008) are reported with green diamonds. 


\section{Cosmological outreach}

Extragalactic radio sources are expected to be the most relevant contaminant of the CMB angular power spectrum for angular scales up to few arcminutes in the $70-100 \mathrm{GHz}$ frequency range [7], where Galactic foregrounds (Galactic synchrotron and thermal dust emissions) are, instead, at a minimum. Subtracting foreground sources from CMB in polarisation at such high frequency is usually based on extrapolations from low frequencies $(\lesssim 20 \mathrm{GHz})$. However, they turn out to be inadequate to model the radio source contribution. Thus, our proposed observations (the ALMA ones, in particular) will improve the characterization of polarised foreground from extragalactic radio sources, allowing proper subtraction of this signal. This, in an era of precision cosmology, will be essential to shed light on primordial B-modes in CMB polarised angular power spectrum, expected to be the smoking gun for inflation in the early universe.

Polarimetric studies like those proposed here are indeed useful also to improve performance of many ground-based facilities looking for primordial B-modes or cosmic polarisation rotation (constrained to be less than $\sim 1^{\circ}$ ) since they can provide suitable calibrators, e.g., to fix at subdegree accuracy scales the observed polarisation angle, currently one of the most limiting aspect in cosmological polarimetric observations [8].

\section{References}

[1] V. Galluzzi and M. Massardi, The polarimetric multi-frequency radio sources properties, International Journal of Modern Physics D 25 (2016) 1640005.

[2] M. Massardi, S. G. Burke-Spolaor, T. Murphy, R. Ricci, M. López-Caniego, M. Negrello et al., A polarization survey of bright extragalactic AT20G sources, MNRAS 436 (Dec., 2013) 2915-2928, [1309.2527].

[3] A. Sajina, B. Partridge, T. Evans, S. Stefl, N. Vechik, S. Myers et al., High-frequency Radio Spectral Energy Distributions and Polarization Fractions of Sources in an Atacama Cosmology Telescope Survey Field, ApJ 732 (May, 2011) 45.

[4] L. Bonavera, M. Massardi, A. Bonaldi, J. González-Nuevo, G. de Zotti and R. D. Ekers, The Planck-ATCA Coeval Observations project: the faint sample, MNRAS 416 (Sept., 2011) 559-566, [1106.0614].

[5] M. Massardi, A. Bonaldi, L. Bonavera, G. De Zotti, M. Lopez-Caniego and V. Galluzzi, The Planck-ATCA Co-eval Observations project: analysis of radio source properties between 5 and 217 GHz, MNRAS 455 (Jan., 2016) 3249-3262, [1511. 02605].

[6] E. M. Sadler, R. Ricci, R. D. Ekers, J. A. Ekers, P. J. Hancock, C. A. Jackson et al., The properties of extragalactic radio sources selected at 20GHz, MNRAS 371 (Sept., 2006) 898-914, [astro-ph/0603437].

[7] G. de Zotti, R. Ricci, D. Mesa, L. Silva, P. Mazzotta, L. Toffolatti et al., Predictions for high-frequency radio surveys of extragalactic sources, A\&A 431 (Mar., 2005) 893-903, [astro-ph/ 0410709 ].

[8] M. Massardi, V. Galluzzi, R. Paladino and C. Burigana, Polarization of extragalactic radio sources: CMB foregrounds and telescope calibration issues, International Journal of Modern Physics D 25 (2016) 1640009. 